\title{
Evidências de Validade dos Indicadores Emocionais no Desenho da Figura Humana
}

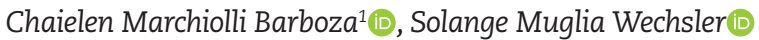 \\ Pontifícia Universidade Católica de Campinas, Campinas-SP, Brasil
}

\section{RESUMO}

O desenho da figura humana (DFH) é um instrumento amplamente utilizado na avaliação psicológica. O objetivo deste estudo foi investigar as evidências de validade dos indicadores emocionais no DFH pelo Sistema de Triagem Emocional (STE). A amostra foi composta por 310 crianças, divididas em grupo regular com 173 crianças, grupo regular com indicadores com 55 crianças e grupo clínico com 82 crianças. Foram utilizados o STE, a Escala Multidimensional de Satisfação de Vida para crianças e de Afeto positivo e negativo. Os resultados demonstraram diferenças significativas para grupos nos indicadores emocionais pelo STE $(F=12,140 ; p \leq 0,01)$. Houve correlações negativas $(p<0,01)$ do STE com a Escala Multidimensional de Satisfação de Vida na dimensão do Self, porém não significativas com a Escala de Afetos. Conclui-se sobre as evidências de validade do STE para avaliar os indicadores emocionais no DFH e distinguir crianças que necessitam de atendimento antes do agravamento dos problemas.

Palavras-chave: personalidade; avaliação psicológica; indicadores emocionais; desenho da figura humana.

\section{ABSTRACT - Validity evidence of the emotional indicators in Human Figure Drawing}

Human figure drawing (HFD) is an instrument widely used in psychological assessment. The aim of this study was to investigate the evidence of validity of emotional indicators in HFD through the Emotional Screening System (ESS). The sample consisted of 310 children, divided into a regular group containing 173 children, a regular group with indicators containing 55 children and a clinical group containing 82 children. The ESS, the Multidimensional Life Satisfaction Scale for children and the Positive and negative affect scale, were used. The results showed significant differences for groups in the emotional indicators of the ESS $(F=12.140 ; p \leq .01)$. There were negative correlations $(p<.01)$ of the ESS with the Multidimensional Scale of Life Satisfaction in the dimension of the Self, but not significant with the Scale of Affections. There was evidence of validity of the ESS to assess the emotional indicators in HFD and to distinguish children who need care before the problems worsen.

Keywords: personality; psychological evaluation; emotional indicators; human figure design.

RESUMEN - Evidencias de Validez de los Indicadores Emocionales en el Diseño de la Figura Humana

El dibujo de la figura humana (DFH) es un instrumento frecuentemente utilizado en la evaluación psicológica. El objetivo de este estudio fue investigar las evidencias de validez de los indicadores emocionales en DFH por el Sistema de Triaje Emocional (STE). La muestra estuvo conformada por 310 niños, divididos en: un grupo regular con 173 niños, un grupo regular con indicadores con 55 niños y un grupo clínico con 82 niños. Se utilizó el STE, la Escala de Satisfacción con la Vida Multidimensional para niños y de Afecto positivo y negativo. Los resultados mostraron diferencias significativas para los grupos en los indicadores emocionales por STE $(F=12.140 ; p \leq 0.01)$. Hubo correlaciones negativas $(p<0.01)$ del STE con la Escala Multidimensional de Satisfacción con la Vida en la dimensión del Self, pero no significativas con la Escala de Afectos. Se concluye sobre las evidencias de validez del STE para evaluar los indicadores emocionales en el DFH y distinguir a los niños que necesitan cuidados antes de que los problemas se agraven. Palabras clave: personalidad; evaluación psicológica; indicadores emocionales; diseño de figuras humanas.

O Desenho da Figura Humana (DFH) é um dos instrumentos mais utilizados na avaliação psicológica tanto para a avaliação cognitiva quanto da personalidade (Villemor-Amaral \&Werlang, 2008; Wechsler \& Nakano, 2012). É também uma ferramenta empregada no psicodiagnóstico infantil, por ser uma atividade lúdica, e uma forma de rapport. A ampla utilização do DFH pode ser atribuída ao fato de ser uma técnica simples, de fácil aplicação e acesso, baixo custo, familiar ao mundo infantil e adequada para crianças que ainda não conseguem verbalizar o que pensam e sentem (Handler \& Thomas, 2014; Oakland, Douglas \& Kane, 2015.

O DFH pode ser compreendido dentre três grandes vertentes teóricas, segundo Arteche e Bandeira (2006): 1. avaliação cognitiva; 2. avaliação projetiva, ou seja, aspectos relacionados ao inconsciente; 3. avaliação emocional, diferente dos aspectos projetivos, pois é utilizada uma análise empírica. Além dessas 
propostas, Oliveira e Wechsler (2016) apontaram que o desenho da figura humana pode ser utilizado como uma forma de compreensão de aspectos criativos presente no desenho, incluindo uma quarta maneira de interpretá-lo. Os estudos sistematizados sobre o DFH inicialmente foram relacionados como medida dos aspectos cognitivos iniciados por Goodenough em 1926 e, posteriormente, por Harris (1963), Koppitz (1984), Naglieri (1988). No âmbito nacional, Sisto (2005) e Wechsler $(2003,2018)$ realizaram estudos e apresentaram evidências de validade, precisão e normas do DFH como sistema de avaliação cognitiva. Embora exista grande parte da literatura indicando as evidências da validade do DFH na avaliação cognitiva, ainda se notam resultados contraditórios sobre a sua utilização para avaliação emocional.

\section{Estudos sobre Avaliação Emocional no DFH}

Os estudos iniciais sobre a avaliação emocional do DFH tiveram enfoque projetivo, baseado na Psicanálise, tendo sido sistematizados, inicialmente, por Machover (1949) que desenvolveu seu trabalho em clínicas e hospitais. A autora enfatizava que cada figura desenhada é particular, contendo significado exclusivo para cada indivíduo (Batista et al., 2014). Como precursora nacional dos aspectos projetivos do DFH, destaca-se Van Kolck que, em 1973, afirmou que o desenho refletia a representação que a criança fazia de si mesmo. Nesse sentido, nos seus estudos com crianças comprometidas emocionalmente e não comprometidas tentou validar os indicadores emocionais no desenho da figura humana. A autora avaliou a presença de ansiedade em 120 crianças entre 7 e 12 anos, utilizando os trinta indicadores emocionais de Koppitz e os correlacionou com os vinte índices de ansiedade proposto por Handler em 1967. O estudo apontou baixas correlações e não foi possível, portanto, a sua validação (Van Kolck, 1974; 1981), demonstrando assim a fragilidade de análise dos indicadores nos desenhos com ênfase projetiva, pois dão margem para grande subjetividade.

Em relação aos aspectos emocionais do DFH, a psiquiatra americana Koppitz observou que os sinais gráficos no DFH poderiam ser vistos tanto da maturidade mental quanto de problemas emocionais. Essa autora acreditava que as crianças representavam no desenho o seu autoconceito (Hasan et al., 2014; Silva, et al., 2015). Por meio de uma lista de indicadores emocionais, estruturou critérios para obter a validade clínica do desenho com o objetivo de diferenciar as crianças com e sem conflitos emocionais. Nesse sistema, é exigida uma frequência de itens menor que $16 \%$ de indicadores para que estes sejam considerados como emocionais, para cada faixa etária (Koppitz, 1968; 1984)

Existem vários estudos internacionais utilizando o Sistema Koppitz. Por exemplo, na pesquisa de Melgarejo et al. (2015), foram avaliados indicadores emocionais do
DFH em crianças vítimas de violência e crianças sem essa condição, e confirmaram que o grupo de crianças vítimas de violência apresentou mais indicadores emocionais do que o grupo controle. Também Zapata e Cifuentes (2017) realizaram uma pesquisa comparando grupo de crianças com diagnóstico de Transtorno de Déficit de Atenção e Hiperatividade (TDAH) com crianças sem o transtorno e observaram que a maior média de indicadores emocionais ocorreu para o grupo de crianças com TDAH. Na Colômbia, Meerbeke et al. (2011) avaliaram a validade do Sistema Koppitz para crianças colombianas, no entanto, não conseguiram níveis satisfatórios de confiabilidade entre os avaliadores e propuseram novas tabelas de pontuação contextualizadas, ressaltando que a correção deve ser feita com cautela, devido ao grande componente subjetivo que existe na pontuação dos critérios de Koppitz.

A utilização do Sistema Koppitz para avaliação dos indicadores emocionais no DFH tem sido utilizada em vários estudos brasileiros. Por exemplo, para identificar a problemática de crianças vítimas de abuso sexual (Cara \& Neme, 2016), na comparação de crianças com outras dificuldades emocionais sem essas condições (Rosa et al., 2019; Santos et al., 2010), de crianças infectadas com vírus HIV (Braghetto, \& Carvalho, 2013), com problemas orgânicos, ou com dificuldades de aprendizagem (Souza \& Hoffman, 2013). Entretanto, a utilização do sistema de Koppitz tem sido questionada (Bandeira \& Arteche, 2008). Vários itens considerados emocionais também são encontrados em crianças de populações não clínicas, como foi observado nos estudos de Bartholomeu et al. (2006), Hutz e Antoniazzi (1995) e de Linhares, et al. (2005). Zortéa et al. (2008) encontraram pouca diferenciação entre crianças institucionalizadas e não institucionalizadas por esse sistema. Existe a proposta de uma análise global dos desenhos por juízes treinados (Segabinazzi \& Bandeira, 2012), embora as evidências de validade desse sistema ainda estejam sendo verificadas). Embora o sistema de Koppitz tenha sido muito utilizado, pois permite maior objetividade nas análises, no entanto, ele ainda é um sistema qualitativo e não permite comparar crianças por meio de tabelas normativas para interpretar os resultados.

\section{Avaliação do DFH por Total de Indicadores Emocionais}

A análise emocional por cada item presente no DFH foi questionada, pois necessita de um amplo conhecimento clínico sobre cada criança para validar sua interpretação. Assim sendo, quase 30 anos após a construção do sistema de Koppitz, os autores Naglieri et al. (1991) propuseram a avaliação pelo total de indicadores do DFH ao invés da análise de item por item. Assim sendo, esses autores combinaram as propostas anteriores de avaliação emocional do DFH por Machover e Koppitz em 
um novo sistema denominado Draw-a-Person: Screening Procedure for Emotional Disturbance Test (DAP:SPED). Nesse sistema, é feita uma avaliação total da pontuação derivada de três desenhos (homem, mulher e si mesmo) auferidos pela presença de itens considerados raros, isto é, com porcentagem menor de $16 \%$ na população. A proposta não é a interpretação dos desenhos, mas sim estabelecer parâmetros para crianças norte-americanas por faixa etária, sendo sugerida uma avaliação emocional mais completa quanto maior for a soma total das pontuações dos três desenhos, sendo assim possível identificar crianças com problemas de conduta ou com necessidades especiais (Naglieri \& Peiffer, 1992; Matto et al., 2005. Vale ressaltar que, tanto Naglieri et al. (1991) como Wechsler (2018) desenvolveram também sistemas para a avaliação cognitiva para o DFH.

O sistema de avaliação do total de indicadores proposto por Naglieri et al., (1991) foi investigado em estudo brasileiro por Wechsler et al. (2011) com crianças de escolas públicas e particulares, sem histórico clínico. Os resultados indicaram que a pontuação total era influenciada pelo sexo da criança, idade, tipo de figura desenhada, tipo de escola e interações. Porém, quando os itens que representavam aspectos de desenvolvimento foram retirados, de acordo o sistema cognitivo de Wechsler (2003), o efeito de idade e tipo de escola foi eliminado. Um Sistema de Triagem Emocional (STE) foi então elaborado por Wechsler (2011), considerando a redução de itens que apresentavam imaturidade cognitiva para determinadas idades.

As evidências de validade do STE foram investigadas em estudos iniciais. Por exemplo, a pesquisa de Comparini e Wechsler (2015) comparou crianças institucionalizadas com outras em clínicas escola e de escolas regulares e constatou que diferenças de grupo e de sexo podiam ser discriminadas pelo STE. Em outro estudo, Comparini et al. (2017) analisaram as relações obtidas pelo STE com aqueles resultantes do teste Youth Self Report (YSR) que busca avaliar problemas de comportamento e competência social em adolescentes, entretanto, não foram encontradas correlações positivas nos totais desses dois instrumentos, apenas para sete itens emocionais.

Posteriormente, Rosa (2018) utilizou o STE e o sistema de Koppitz para comparar crianças de grupo clínico com Transtorno de Déficit de Atenção e Hiperatividade (TDAH), com outros grupos de crianças com dificuldades de aprendizagem e de crianças que não apresentavam essas condições. O sistema de Koppitz e o STE demonstraram estar significativamente correlacionados, porém o STE demonstrou ser mais sensível, pois identificou significativamente o grupo clínico, o que não ocorreu com o sistema de Koppitz, indicando assim evidências de validade para o STE. Tais dados indicam a necessidade de mais pesquisas sobre a validade de sistemas para avaliação emocional do DFH.
Os trabalhos feitos no país confirmam os esforços dos pesquisadores na procura de sistemas com qualidades científicas para avaliar o desenho da figura humana como medida emocional, tarefa ainda não conseguida segundo a relação de testes aprovados pelo Sistema de Avaliação de Testes Psicológicos (SATEPSI). Vale ressaltar que, segundo as regulamentações do Conselho Federal de Psicologia (2018), todo teste psicológico a ser utilizado no país deve apresentar as qualidades psicométricas do instrumento, ou seja, evidências de validade, precisão e normas atualizadas, abrangendo não só os testes objetivos, mas também os projetivos para que sejam considerados aprovados para uso profissional. As evidências de validade podem ser obtidas por diversos métodos, sendo um deles a comparação de um construto específico com variáveis ou critérios externos, o que pode ser feito por grupos contrastantes ou pela comparação por divergência ou convergência com outro teste que avalie construtos diferentes ou semelhantes (American Educational Research Association, American Psychological Association, National Council of Measurement in Education, 2014).

Considerando, portanto, a necessidade de mais pesquisas que sejam capazes de verificar a validade do DFH enquanto instrumento de medida emocional, este estudo visou investigar as evidências de validade do STE comparado com medidas externas para discriminar grupos contrastantes (clínico e não clínicos). Além disso, considerando que os instrumentos em Psicologia Positiva visam avaliar aspectos da saúde mental, este estudo também analisou se haveria correlações negativas entre o STE com a Escala Multidimensional de Satisfação de Vida para Crianças e a Escala de Afeto Positivo e Negativo.

\section{Método}

\section{Participantes}

A amostra foi composta por 310 crianças, 172 do sexo feminino e 138 do sexo masculino, com idades de 9 a 12 anos, conforme apresentado na Tabela 1 .

A amostra foi selecionada por conveniência e divididas em três grupos distintos conforme os seguintes critérios: grupo Regular ou sem problemas continha 173 crianças sendo $58 \%$ do sexo feminino e $42 \%$ do sexo masculino que frequentavam o Ensino Fundamental de escola pública; o grupo Regular com indicadores foi composto por 55 crianças, $62 \%$ do sexo feminino e $38 \%$ do sexo masculino que frequentavam escola pública e foram indicadas pelos responsáveis como portadoras de problemas de comportamento, mas não passavam por atendimento psicológico; e o grupo Clínico formado por 82 crianças, $45 \%$ do sexo feminino e $55 \%$ do sexo masculino e eram atendidas por duas clínicas particulares do estado de São Paulo, provenientes tanto de escola pública quanto de escola particular. 
Tabela 1

Médias e Desvio Padrão Idades dos Participantes

\begin{tabular}{|c|c|c|c|}
\hline Variável Dependente & Grupo & Média & Desvio Padrão \\
\hline \multirow{3}{*}{ Idade } & Clínico & 9,976 & 0,9023 \\
\hline & Regular indicadores & 9,727 & 0,7317 \\
\hline & Regular & 9,798 & 0,7847 \\
\hline
\end{tabular}

\section{Instrumentos}

Desenho da Figura Humana - Sistema de Triagem Emocionais - STE (Wechsler, 2011). O Sistema de Triagem Emocional (STE) está baseado nos totais de indicadores considerados emocionais presentes no DFH, e a aplicação é para crianças entre 6 a 12 anos de idade. Essa escala foi inicialmente baseada no sistema de Naglieri et al. (1991), tendo sido posteriormente adaptada, pois vários itens são pontuados apenas a partir de determinada faixa etária, sendo então considerados como indicadores de imaturidade cognitiva, segundo o sistema cognitivo de Wechsler (2003). Por exemplo, no aspecto desintegração "braço-tronco" é considerado como indicador crianças a partir de 7 anos; o aspecto omissão "nariz" e "pés" são considerados como indicadores crianças a partir de 8 anos de idade; omissão de "mãos" a partir de 9 anos de idade; e omissão de "dedos" a partir de 10 anos de idade.

Nesse sistema, as crianças executam dois desenhos, um da figura feminina e um da figura masculina, independentemente da ordem. A correção é composta por 59 itens que são avaliados tanto para o desenho da figura masculina quanto da feminina e, ao final, são avaliados os totais obtidos na soma das duas figuras. Os itens são agrupados em 16 dimensões e avaliados em presença/ausência, ou seja, pontuação 0 para ausência e 1 para presença. As dimensões são: 1. tamanho da figura; 2. distância das margens; 3. posição; 4. desintegração; 5. qualidade do traçado; 6. sombreamentos: 7. tipo de figura; 8. contexto; 9. omissões; 10. desproporção; 11. nudez; 12. transparências; 13 . olhos; 14 . boca; 15 . braços e 16 . dedos.

Algumas pesquisas com o STE já indicaram evidências de sua validade (Comparini \& Wechsler, 2015; Rosa, 2018), pois foi possível discriminar grupos clínicos e não clínicos, como já relatado anteriormente. A precisão de correção por esse sistema também já foi analisada por Ferreira (2011), que obteve correlações entre juízes variando de $r=0,63$ a 0,90 para a figura masculino e feminina.

Escala Multidimensional de Satisfação de Vida para Crianças (EMSVC) (Giacomoni, \& Hutz, 2008). A EMSVC é uma escala que engloba aspectos do dia a dia da criança, entre 7 e 12 anos de idade. O instrumento é composto por 50 itens do tipo Likert entre 1 a 5 seguindo os critérios: 1 - Nem um pouco; 2 - Um pouco; 3 - Mais ou menos; 4 - Bastante; 5 - Muitíssimo. É respondido pela própria criança e o tempo de aplicação é normalmente de 30 minutos. Segundo estudos de Giacomoni e Hutz (2008), a escala possui seis fatores que representam domínios de satisfação de vida infantil, tais como: Self (eu mesmo), Self comparado (eu amizade), Não violência, Família, Amizade e Escola. O fator Self está relacionado às características que a criança faz de si mesmo, como autoestima, bom-humor e demonstração de afeto. O Self comparado agrupa itens em que a criança compara características dos seus pares, ou seja, relacionado ao lazer, amizade e desejos. $O$ fator Não violência é associado a ausência de comportamento agressivo. O fator Família é relacionado ao ambiente familiar satisfatório. A Amizade mostra características das relações e o nível de satisfação. E o fator Escola apresenta o ambiente escolar e os relacionamentos interpessoais dentro desse ambiente.

Existem ainda poucos estudos sobre a validade desse instrumento. Os estudos de validade realizados por Giacomoni e Hutz (2008) com 661 crianças de escolas regulares apontaram a existência de seis fatores que representam domínios de satisfação de vida infantil: Self (eu mesmo), Self comparado (eu amizade), Não violência, Família, Amizade e Escola. A comparação desses fatores com variáveis externas indicou correlações positivas significativas com medidas de autoestima $(r=0,37$ a 0,51$)$ e negativas com ansiedade $(r=-0,16$ a $-0,51)$. A precisão das subescalas apresentou índices de fidedignidade satisfatórios, variando de 0,82 a 0,86 , com exceção da escala de violência, que obteve índice de 0,66.

Escala de Afeto Positivo e Negativo (Giacomoni, \& Hutz, 2006). A escala é composta por 34 itens, de afetos positivos e negativos, com respostas do tipo Likert de 5 pontos, que variam de 1 a 5 (1 - Nem um pouco; 2 - Um pouco; 3 - Mais ou menos; 4 - Bastante; 5 - Muitíssimo), indicando o quanto as crianças estão se sentindo de acordo com cada item. A aplicação é para crianças de 7 a 12 anos de idade. Como relatado na construção desse instrumento, as evidências de validade de sua estrutura interna foram feitas em estudo com 661 crianças de escolas regulares. O resultado pela análise fatorial indicou a existência de dois fatores, um classificado como afeto positivo e outro como negativo.

As evidências com variáveis externas foram analisadas por meio da comparação com outras escalas que mediam construtos semelhantes (Escala de Autoestima e Satisfação com a Vida) ou divergente (Inventário de Depressão Infantil (CDI), Inventário de Ansiedade 
Traço-Estado). Correlações positivas e significativas foram encontradas entre construtos semelhantes $(r=0,45)$ e com construtos divergentes $(r=-0,35)$, indicando assim a sua validade. Também a precisão das escalas pelo coeficiente alfa de Cronbach foi satisfatória atingindo para o Afeto Positivo $(0,89)$ e o Afeto Negativo $(0,89)$. França-Freitas, Del Prette e Del Prette (2017) também encontrou relações significativas entre satisfação com a vida e bem-estar subjetivo em crianças dotadas e talentosas.

Ficha de Identificação da Criança. Foi desenvolvida neste estudo e preenchida pelos pais das crianças, visando investigar a história clínica desta, ou seja, se estava ou tinha passado por tratamento psicoterápico ou psiquiátrico. Esse instrumento teve como objetivo auxiliar na discriminação dos grupos de estudo.

\section{Procedimentos}

Após a aprovação do projeto de pesquisa pelo Comitê de Ética em Pesquisa com seres humanos $\mathrm{n}^{\circ}$ 62463516.9.0000.5481, realizou-se o contato com a direção das escolas para explicação dos objetivos da pesquisa. Nessa ocasião, foram definidas as classes educacionais de acordo com a idade das crianças para a pesquisa. Para os pais, foram enviados os Termo de Consentimento Livre e Esclarecido assim como a Ficha de Identificação.

A aplicação da pesquisa ocorreu de forma individual nas próprias escolas, em salas vazias que estavam disponíveis ou biblioteca. Os colaboradores foram estudantes de Psicologia, do $3^{\circ}$ ao 50 anos atuaram como ajudantes de pesquisa voluntários para administrar e corrigir os instrumentos. $\mathrm{Na}$ aplicação nas clínicas de Psicologia, o responsável pela coleta foi o terapeuta da criança, que também recebeu orientação do procedimento. A criança primeiramente fez os dois desenhos (figura feminina e masculina), na ordem de sua preferência, de acordo com as instruções existentes no STE. O examinador lia as perguntas das escalas de satisfação com a vida e de afeto quando a criança apresentava dificuldade de leitura.

Com os instrumentos aplicados, foi iniciada a correção dos testes de acordo com o sistema proposto por cada um, ou seja, o DFH para cada figura foi analisado conforme o Sistema Triagem Emocional (STE) proposto por Wechsler (2011). A correção da escala EMSVC seguiu os critérios descritos por Giacomoni e Hutz (2008) e a Escala de Afeto Positivo e Negativo foi segundo a proposta de Giacomoni e Hutz (2006).

\section{Análise de Dados}

A Análise Multivariada da Covariância (MANCOVA) foi utilizada para analisar o total de indicadores emocionais na figura feminina e masculina segundo o STE para analisar o efeito do sexo da criança, tipo de grupo (Clínico, Regular com indicadores e Regular), e Idade (covariável). Quando os resultados eram significativos, estes foram também analisados pela ANOVA o efeito calculado por (Maior raiz de Roy). Posteriormente foi utilizada a correlação de Pearson para comparar os resultados do STE para a figura feminina e masculina com os resultados da Escala de Satisfação de Vida e de Afetos Positivos e Negativos.

\section{Resultados}

Primeiramente foram analisados os totais dos indicadores emocionais do DFH por meio do Sistema de Triagem Emocional (STE), comparando os grupos, Clínico, Regular com indicadores e Regular. Na Tabela 2, serão apresentadas as médias totais da figura feminina e masculina no DFH de acordo com o sexo da criança.

Tabela 2

Médias Totais na Figura Feminina e Figura Masculina segundo o STE

\begin{tabular}{|c|c|c|c|c|}
\hline Variável Dependente & Sexo & Grupo & Média & Erro Padrão \\
\hline \multirow{6}{*}{ Figura Feminina Total } & \multirow{3}{*}{ Feminino } & Clínico & 5,973 & 0,463 \\
\hline & & Regular Problema & 4,520 & 0,438 \\
\hline & & Regular & 4,195 & 0,281 \\
\hline & \multirow{3}{*}{ Masculino } & Clínico & 6,825 & 0,367 \\
\hline & & Regular Problema & 5,873 & 0,737 \\
\hline & & Regular & 4,271 & 0,422 \\
\hline \multirow{6}{*}{ Figura Masculina Total } & \multirow{3}{*}{ Feminino } & Clínico & 5,994 & 0,498 \\
\hline & & Regular Problema & 3,836 & 0,471 \\
\hline & & Regular & 4,064 & 0,302 \\
\hline & \multirow{3}{*}{ Masculino } & Clínico & 7,304 & 0,395 \\
\hline & & Regular Problema & 5,245 & 0,794 \\
\hline & & Regular & 4,883 & 0,455 \\
\hline
\end{tabular}


Conforme a Tabela 2, na figura feminina total, o grupo Clínico apresentou médias maiores tanto no sexo feminino $(M=5,973 ; D P=0,463)$ quanto no sexo masculino $(M=6,825 ; D P=0,367)$. Na figura masculina total, o grupo Clínico também teve médias superiores tanto no sexo feminino $(M=5,994 ; D P=0,498)$ como no sexo masculino $(M=7,304 ; D P=0,395)$. A análise por meio da MANCOVA demonstrou que a variável Grupo obteve diferença significativa em todos os testes realizados $(F=12,140 ; p \leq 0,01)$. Para verificar melhor essas diferenças de grupo, foi realizado a ANOVA, conforme apresentado na Tabela 3, a seguir.

Tabela 3

Análise da Variância para os totais das figuras femininas e masculinas no STE

\begin{tabular}{lcccccc}
\hline \multicolumn{1}{c}{ Origem } & Variável dependente & SSQ & GL & QM & $F$ & Eta Parcial Quadrado \\
\hline \multirow{2}{*}{ Idade } & Fig Fem Total & 0,17 & 1 & 0,17 & 0,05 & 0,00 \\
& Fig Masc Total & 0,00 & 1 & 0,00 & 0,00 & 0,00 \\
\multirow{3}{*}{ Sexo } & Fig Fem Total & 3,07 & 1 & 3,07 & 0,98 & 0,01 \\
& Fig Masc Total & 17,87 & 1 & 17,87 & $4,90^{*}$ & 0,04 \\
\multirow{2}{*}{ Grupo } & Fig Fem Total & 59,43 & 2 & 29,71 & $9,46^{* * *}$ & 0,22 \\
& Fig Masc Total & 69,67 & 2 & 34,83 & $9,56^{* * *}$ & 0,25 \\
\multirow{2}{*}{ Sexo* Grupo } & Fig Fem Total & 4,22 & 2 & 2,11 & 0,67 & 0,01 \\
& Fig Masc Total & 0,53 & 2 & 0,26 & 0,07 & 0,01 \\
\hline
\end{tabular}

Nota. ${ }^{*} p \leq 0,05 ;{ }^{* *} p \leq 0,01 ;{ }^{* * *} p \leq 0,001$

Conforme os dados apresentados na Tabela 3, houve diferenças significativas no tipo de grupo tanto no total da figura feminina $(F=9,466 ; p \leq 0,001)$ quanto na figura masculina $(F=9,568 ; p \leq 0,001)$. Deve-se notar que o efeito do grupo foi fortemente observado (eta parcial quadrado $=0,22$ a 0,25 ). A variável Sexo exerceu influencia apenas no total da figura masculina $(F=4,908 ; p \leq 0,05)$.

A seguir, com o objetivo de verificar a influência do tipo de grupo, sexo e idade na Escala Multidimensional de Satisfação de Vida foi feita a MANCOVA. Os resultados obtidos demonstraram diferenças significativas na variável sexo $(F=2,195 ; p \leq 0,05)$. Para verificar tais diferenças, foi realizada a análise ANOVA. Foi possível verificar o efeito significativo na variável idade na área Escola $(F=7,201 ; p \leq 0,01)$, na variável sexo na área denominada Self $(F=4,208 ; p \leq 0,05)$ e Família $(F=4,362 ; p \leq 0,05)$. Deve se destacar que a variável Grupo não apresentou diferenças significativa em nenhuma das dimensões da Escala Multidimensional de Satisfação de Vida para Crianças.

A seguir, foram analisados os efeitos das variáveis Grupo, Sexo e Idade nos afetos positivos e negativos. Na análise MANCOVA, não houve efeito de grupo, mas a interação de sexo/grupo apresentou diferença significativa $(F=3,708 ; p \leq 0,05)$. A ANOVA indicou que somente no fator de Afeto negativo pode-se observar que a interação sexo-grupo teve influência significativa $(F=3,367$; $p \leq 0,05)$, tendo o sexo feminino maiores médias no grupo Regular.

A seguir, na Tabela 4, foram analisadas as relações entre os totais dos indicadores emocionais do DFH por tipo de figura, segundo o STE, com os totais da Escala Multidimensional com a Satisfação com a vida e da Escala de Afeto Positivo e Negativo, pela Correlação de Pearson.

Tabela 4

Correlação de Pearson entre Totais no STE por Figura com as Escala Multidimensional de Satisfação de Vida para Criança e a Escala de Afeto Positivo e Negativo

\begin{tabular}{lcc}
\hline \multicolumn{1}{c}{ Variáveis } & Figura Feminina Total & Figura Masculina Total \\
\hline Self & $-0,174^{* *}$ & $-0,176^{* *}$ \\
Self Comparado & $-0,080$ & $-0,041$ \\
Não Violência & $-0,029$ & $-0,044$ \\
Família & $-0,079$ & $-0,097$ \\
Socialização & $-0,074$ & $-0,100$ \\
Escola & $-0,161^{* *}$ & $-0,173^{* *}$ \\
Afeto Positivo & $-0,033$ & $-0,027$ \\
Afeto Negativo & $-0,027$ & $-0,054$ \\
\hline
\end{tabular}

Nota. ${ }^{*} p \leq 0,05 ;{ }^{* *} p \leq 0,01$ 
Segundo os dados apresentados na Tabela 4, houve correlações significativas, no sentido esperado negativo pelo STE, tanto da figura feminina $(r=-0,174)$ quanto da feminina $(r=-0,176)$ com a dimensão Self da Escala Multidimensional de Satisfação com a Vida. Nas demais variáveis dessa escala, não foram encontradas correlações significativas nem com as aquelas avaliadas pela Escala de Afeto Positivo e Negativo.

\section{Discussão}

O desenho da figura humana é uma das técnicas mais utilizadas por psicólogos internacionalmente para avaliação cognitiva e também emocional da criança (Oakland et al., 2016; Handler \& Thomas, 2014). Embora exista consenso na literatura nacional sobre a validade do DFH como medida cognitiva (Sisto, 2005; Wechsler, 2003; 2018) ainda existe controvérsias sobre o uso do DFH para medida de indicadores emocionais (Rosa et al., 2019).

Dentre os sistemas mais conhecidos internacionalmente para avaliar os indicadores emocionais na figura humana, encontram-se os de Koppitz (1968), que interpreta item por item, e o de Naglieri et al. (1991), que se propõe a fazer uma análise total dos itens afim de comparar a criança com outras da sua faixa etária, visando assim o encaminhamento para uma avaliação mais completa emocional, caso necessário. No Brasil, o sistema de Koppitz tem sido utilizado em vários estudos, mas recebeu questionamento por não exibir níveis satisfatórios de precisão entre avaliadores e por apresentar itens que estão também presentes em crianças de populações não clínicas (Bartholomeu et al., 2006; Linhares et al., 2005; Hutz, \& Antoniazzi, 1995; Zortea et al., 2008). O sistema de Naglieri, por sua vez, demonstrou ser influenciado pelo sexo da criança, sua idade, tipo de figura e escola, no estudo realizado por Wechsler et al. (2011). Uma avaliação global dos indicadores emocionais por juízes também tem sido estudada por Segabinazzi e Bandeira (2012), embora ainda em estudos de validade.

Uma adaptação do sistema de Naglieri foi realizada no país por Wechsler (2011), com a retirada de itens que apresentavam imaturidade cognitiva, baseando-se nas suas pesquisas com avaliação cognitiva no DFHIII (Wechsler, 2003). As evidências de validade desse método, denominado Sistema de Triagem Emocional (STE), foram analisadas neste estudo. Duas estratégias foram utilizadas: a investigação se os indicadores emocionais no STE poderiam distinguir grupos contrastantes (Clínico, Regular com indicadores e Regular) e a comparação com variáveis externas, ou seja, dois instrumentos da Psicologia Positiva que deveriam estar negativamente relacionados com os resultados do STE (Escala Multidimensional de Satisfação com a Vida e a Escala de Afeto Positivo e Negativo).
Os resultados dos totais de indicadores emocionais no desenho da figura humana pelo STE indicaram que esse sistema pode fazer uma forte discriminação entre os grupos, ou seja, é possível discriminar crianças com indicadores emocionais para uma avaliação mais completa, antes do agravamento dos problemas emocionais. Foi demonstrado que o grupo clínico possuía mais indicadores emocionais do que outros grupos, ou seja, maiores pontuações das crianças que estavam passando por processo terapêutico e que apresentam problemas emocionais do que aquelas consideradas como não clínicas, demonstrando assim a evidência de validade do STE. Portanto, o STE demonstrou ser bastante sensível para triagem emocional, confirmando o que já tinha sido constatado por Rosa (2018) ao comparar crianças com TDAH com outros grupos de crianças de crianças sem essas condições e no estudo de Comparini e Wechsler (2015), ao observar que o STE podia distinguir crianças institucionalizadas de outras em clínicas escola e de crianças regulares.

A relação dos indicadores emocionais no DFH com a Escala Multidimensional de Satisfação de Vida para Crianças apresentou, por sua vez, relação significativa negativa no fator Self (eu mesmo), tanto para a figura feminina quanto para a masculina. Essa direção invertida confirma que a problemática emocional pode afetar a autoestima e o bom-humor, variáveis importantes na avaliação do bem-estar subjetivo. Entretanto, não houve relações significativas do STE com as demais dimensões desse instrumento, não confirmando, portanto, nossa hipótese. Deve ser ressaltado, entretanto, que são pouco os estudos sobre as evidências de validade da Escala Multidimensional de Satisfação da Vida, tal como relatadas por Giacomoni e Hutz (2008). Assim sendo, as evidências de validade do STE foram confirmadas parcialmente.

Em relação à Escala de Afeto Positivo e Negativo, embora tenham existidas correlações nas direções esperadas, não houve relações significativas com o STE. Apenas na interação sexo/grupo houve resultado significativo, para o sexo feminino. Segundo Giacomo e Hutz (2006), essa escala teve relação divergente com depressão infantil e ansiedade, o que não ocorreu neste estudo com os indicadores do STE, porém, deve ser ressaltado que essa escala não conseguiu discriminar os tipos de grupos Clínico, Regular com indicadores e Regular, tendo o mesmo acontecido com a Escala Multidimensional de Satisfação da Vida. A escassa literatura sobre essas escalas, entretanto, não permitem conclusões, indicando a necessidade de mais pesquisas sobre suas medidas.

Em conclusão, este estudo demonstrou que o STE possui evidências de validade para discriminar grupos clínicos, porém ainda precisando de mais estudos sobre suas relações com outras variáveis associadas à Psicologia Positiva. Deve também ser considerado que as medidas de indicadores emocionais e bem-estar estão sendo feitas por métodos diferentes, ou seja, expressão por desenho 
e autorrelato. Estudos futuros deverão ser feitos comparando as mesmas formas de expressão para melhor compreensão do funcionamento psicológico infantil. Também serão necessárias outras pesquisas com amostras maiores de grupos clínicos, de outras regiões e realidades socioeconômicas e culturais, para melhor investigação dos indicadores emocionais. Normas para efeitos de triagem emocional por idade deverão ser posteriormente elaboradas a fim de que o STE possa ser utilizado segundo a sua finalidade.

Muitas crianças não são percebidas sobre a intensidade da frequência dos seus distúrbios emocionais. Esse método pode verificar, de forma rápida por ser de aplicação grupal, crianças com necessidade de atendimento, nas escolas, ou instituições, devendo assim ser encaminhadas antes do agravamento de suas problemáticas emocionais. Portanto, o STE traz uma grande contribuição para a área.

Deve-se também salientar que o STE é uma proposta de triagem e que é necessário a utilização de outras técnicas, tais como entrevista, observação assim como outros instrumentos psicológicos que possam complementar a avaliação psicológica. Denota-se a importância de estudos sobre a qualidade científica dos testes psicológicos a fim de que o psicólogo possa utilizar instrumentos validados e confiáveis para a realização do psicodiagnóstico, tendo assim compreensão da dinâmica psíquico infantil.

\section{Agradecimentos}

Os autores agradecem ao financiamento do Conselho Nacional de Desenvolvimento Cientifico e
Tecnológico (CNPQ) pela realização desta pesquisa.

\section{Financiamento}

Todas as fontes de financiamento para elaboração e produção do estudo (coleta, análise e interpretação dos dados, bem como, escrita dos resultados no presente no manuscrito) foram fornecidas pelo projeto de pesquisa ‘130595/2016-0 CNPQ.

\section{Contribuições dos autores}

Declaramos que todos os autores participaram da elaboração do manuscrito. Especificamente, o(s) autor(es) Chaielen Marchiolli Barboza participou(ram) da redação inicial do estudo - conceitualização, investigação, visualização, o(s) autor(es) Chaielen Marchiolli Barboza e Solange Muglia Wechsler participou(ram) da análise dos dados, e o(s) autor(es) Chaielen Marchiolli Barboza e Solange Muglia Wechsler participou(ram) da redação final do trabalho - revisão e edição.

\section{Disponibilidade dos dados e materiais}

Todos os dados e sintaxes gerados e analisados durante esta pesquisa serão tratados com total sigilo devido às exigências do Comitê de Ética em Pesquisa em Seres Humanos. Porém, o conjunto de dados e sintaxes que apoiam as conclusões deste artigo estão disponíveis mediante razoável solicitação ao autor principal do estudo.

\section{Conflito de interesses}

Os autores declaram que não há conflitos de interesses.

\section{Referências}

American Educational Research Association - AERA, American Psychological Association - APA, and National Council on Measurement in Education -NCME. (2014). Standards for Educational and Psychological Testing. AERA Publications Sales

Arteche, A. X., \& Bandeira, D. R. (2006). O desenho da figura humana: Revisando mais de um século de controvérsias. Revista Iberoamerica de Diagnóstico y Evaluación Psicológica, 22(2), 33-156. http://www.redalyc.org/articulo.oa?id=459645449008.

Bandeira, D. R., \& Arteche, A. (2008). Desenho da figura humana. Em A. E. Villemor-Amaral, \& B. S. G. Werlang. (Orgs.). Atualizações em Métodos Projetivos para Avaliação Psicológica (pp. 205-223). Casa do Psicólogo.

Bartholomeu, D., Sisto, R., \& Rueda, F. (2006). Dificuldades de aprendizagem na escrita e características emocionais de crianças. Psicologia em Estudo, 11(1), 139-146. https://www.scielo.br/j/pe/a/XxZdXPnTC8WmQG5mWCpp58g/?lang=pt\&format=pdf

Batista, M. A., Sisto, F. F., \& Oliveira, S. M. S. S. (2014). Indicadores de ansiedad en el DFH y rasgos de personalidad en niños: Un estudio de validez. Psicología desde el Caribe, 31(3), 417-434. http://dx.doi.org/10.14482/psdc.31.3.5043

Braghetto, A. C. M., \& Carvalho, A. M. P. (2013). Desempenho escolar, comportamental e desenvolvimento cognitivo e emocional de crianças infectadas pelo HIV: Estudo preliminar. Revista Enfermagem UERJ, 21(1), 29-33. https://www.e-publicacoes.uerj.br/index.php/ enfermagemuerj/article/view/6343

Cara, A. T., \& Neme, C. M. B. (2016). Estudo documental de crianças vítimas de violência sexual: Avaliação dos indicadores de comprometimento emocional segundo Koppitz. Boletim Academia Paulista de Psicologia, 36(91), 383-399. http://pepsic.bvsalud.org/ scielo.php?script $=$ sci_arttext\&pid=S1415-711X2016000200009

Comparini, I., \& Wechsler, S. M. (2015). Indicadores emocionais no desenho da figura humana em grupos contrastantes. Trabalho apresentado no $V$ Congresso de Avaliação Psicológica, São Paulo, Brasil.

Comparini, I., Wechsler, S. M., \& Machado, W. L. (2017). Indicadores emocionais no desenho da figura humana: Investigando evidências de validade. Revista Psicologia: Teoria e Prática, 19(3), 256-269. http://dx.doi.org/10.5935/1980-6906/psicologia.v19n3p256-269

Ferreira, G. V. (2011). Teste projetivo do desenho da figura humana: estudo de fidedignidade entre juizes. [Dissertação de mestrado, Universidade Católica do Rio Grande do Sul]. Universidade Católica do Rio Grande do Sul. http://tede2.pucrs.br/tede2/handle/tede/758 
França-Freitas, M. L. P. F., Del Prette, A., \& Del Prette, Z.A.P. (2017). Habilidades sociais e bem-estar subjetivo em crianças dotadas e talentosas. Psico-USF, 22(1), 1-12. https://doi.org/10.1590/1413-82712017220101

Giacomoni, C. H., \& Hutz, C. (2006). Escala de Afeto Positivo e Negativo para crianças: Estudos de construção e validação. Revista semestral da Associação Brasileira de Psicologia Escolar e Educacional ABRAPEE, 10(2), 235-245. https://doi.org/10.1590/S1413-85572006000200007

Giacomoni, C. H., \& Hutz, C. (2008). Escala Multidimensional de satisfação de vida para crianças: Estudos de construção e validação. Estudos de Psicologia, 25(1), 23-35. https://doi.org/10.1590/S0103-166X2008000100003

Harris, D. B. (1963). Children's drawings as measure of intelectual maturity. Harcourt, Brace \& World.

Hasan, S., Rauf, U., \& Begum, N. (2014). Emotional indicators on human figure drawing test of mentally retarted children with and without hyperactivity. Bahria Journal of Professional Psychology, 13(2), 17-41. https://www.researchgate.net/publication/344402645_Emotional_ Indicators_on_Human_Figure_Drawing_Test_of_Mentally_Retarded_Children_with_and_without_Hyperactivity

Handler, L., \& Thomas, A. D. (2014). Drawings in assessment and psychotherapy: research and application. Routledge/Taylor \& Francis Group.

Hutz, C., \& Antoniazzi, A. S. (1995). O desenvolvimento do desenho da figura humana em crianças de 5 a 15 anos de idade: Normas para avaliação. Psicologia: Reflexão e Crítica, 8(1), 3-18. http://pepsic.bvsalud.org/scielo.php?script=sci_nlinks\&ref=3518575\&pid=S1415711X201500010000600019\&lng=pt

Koppitz, E. M. (1968). Psychological evaluation of children's human figure drawings. Grune \& Stratton.

Koppitz, E. S. (1984). Psychological evaluation of human figure drawings by middle school pupils. Grune \& Stratton.

Linhares, M., Chimello, J., Bordin, M. Carvalho, A., \& Martinez, F. (2005). Desenvolvimento psicológico na fase escolar de crianças nascidas pré-termo em comparação com crianças nascidas a termo. Psicologia, Reflexão e Critica, 18(10), 109-117. https://doi.org/10.1590/ S0102-79722005000100015

Machover, K. (1949). Personality projection in the drawing of the human figure: A method of personality investigation. Charles C Thomas Publisher.

Matto, H., Naglieri, J., \& Clausen, C. (2005). Validity of the Draw-A-Person: Screening Procedure for Emotional Disturbance (DAP:SPED) in Strengths-Based Assessment. Research on Social Work Practice, 15(1), 41-46. https://doi.org/10.1177/1049731504269553

Melgarejo, D. A. L., Melgarejo, O. J., Quevedo, V. S. U., Suhurt, C. H. A., \& Núñez, M. M. R. (2015). Test del dibujo de la figura humana en pacientes en edad pediátrica víctimas de violencia psicológica: estudio de casos. Memorias del Instituto de Investigaciones en Ciencias de la Salud, 13(3), 88-95. https://pesquisa.bvsalud.org/portal/resource/pt/biblio-869054

Meerbeke, A. V., Sandoval-Garcia, C., Ibáñez, M., Talero-Gutiérrez, C., Fiallo, D., \& Halliday, K. (2011). Validation study of human figure drawing test in a colombian school children population. The Spanish Journal of Psychology, 14(1), 464-477. https://doi.org/10.5209/ rev_sjop.2011.v14.n1.42

Naglieri, J. A. (1988). DAP Draw a person: a quantitative scoring system. The Psychological Corporation.

Naglieri, J. A., \& Peiffer, S. (1992). Performance of disruptive behavior disordered and normal samples on the Draw a Person: Screening procedure for emotional disturbance. Psychological Assessment, 4(2), 156-159. https://doi.org/10.1037/1040-3590.4.2.156

Naglieri, J. A., Mcneish, T. J., \& Bardos, A. N. (1991). DAP-SPED: Draw a person: screening procedure for emotional disturbance. Pro-Ed.

Oakland, T., Douglas, S., \& Kane, H. (2015). Top ten standardized tests used internationally with children by school psychologists in 64 countries: a 24-year follow up study. Journal of Psychoeducational Assessment, 34(2), 1-11. https://doi.org/10.1177/0734282915595303

Oliveira, K. S., \& Wechsler, S. M. (2016). Indicadores de criatividade no desenho da figura humana. Psicologia: ciência e profissão, 36(1), 6-19. https://doi.org/10.1590/1982-3703001682014

Resolução No009, de 25 de abril de 2018. Estabelece diretrizes para a reavaliação de Avaliação Psicológica no exercício profissional da psicóloga e do psicólogo, regulamenta o Sistema de Avaliação de Testes Psicológicos - SATEPSI e revoga as Resoluções n ${ }^{\circ}$ 002/2003, n 006/2004 e n $n^{\circ}$ 005/2012 e Notas Técnicas no 01/2017 e 02/2017. Brasília, DF: Conselho Federal de Psicologia.

Rosa, H. R. (2018). Desenho da Figura Humana em crianças: Indicadores emocionais, evidências de validade e precisão. [Tese de Livre Docência, Instituto de Psicologia da Universidade de São Paulo]. Universidade de São Paulo. https://teses.usp.br/teses/disponiveis/livredocencia/47/tde25042019-102736/pt-br.php

Rosa, H. R., Belizario, O. G., Alves, I. C. B., \& Valente, M. L. L. C. (2019). Koppitz's emotional indicators and the Human Figure Drawing: A comparison between a clinical and a school sample. Avances en Psicología Latinoamericana, 37(2), 195-204. http://dx.doi.org/10.12804/ revistas.urosario.edu.co/apl/a.5118

Santos, B. C. A., Ribeiro, M. C. C., Ukita, G. M., Pereira, M. P., Duarte, W. F., \& Custódio, E. M. (2010). Características emocionais e traços de personalidade em crianças institucionalizadas e não institucionalizadas. Boletim de Psicologia, LX(133), 139-152. http://pepsic. bvsalud.org/scielo.php?script=sci_arttext\&pid=S0006-59432010000200002

Segabinazzi, J. D., \& Bandeira, D. R. (2012). Desenho da figura humana para avaliação emocional de crianças: evidências de validade de escalas globais. Em S. M. Wechsler \& T. C. Nakano (Orgs.), O desenho infantil: forma de expressão cognitiva, criativa e emocional. (pp. 149175). Casa do Psicólogo.

Silva, M. C. R., Montiel, J. M., Fiamenghi Jr., G. A., \& Bartholomeu, D. (2015). Técnicas Gráficas aplicadas à Educação e à Saúde. Memnon.

Sisto, F. F. (2005). Desenho da figura humana. Escala Sisto. Editora Vetor.

Souza, J., \& Hoffman, M. S. (2013). Desenho da figura humana: avaliação cognitiva e indicadores de problemas emocionais em crianças. Revista Caminhos, 4(6), 149-160. http://www.scielo.org.co/scielo.php?script=sci_nlinks\&pid =S1794-4724201900020019500017\&lng=en

Villemor-Amaral, A. E., \& Werlang, B. S. G. (2008) (Eds.). Atualizações em Métodos Projetivos para Avaliação Psicológica (pp. 205-223). Casa do Psicólogo.

Van Kolck, O. L. (1974). Correlações entre índices de ansiedade e perturbações emocionais nos desenhos de figuras humanas de crianças. Boletim de psicologia, 25(66),7-12.

Van Kolck, O. L. (1981). Os desenhos no assessoramento psicológico de cardiopatas. Boletim de Psicologia, 33(81), 82-93.

Zapata, E. A. M., \& Cifuentes, V. V. (2017). Indicadores Emocionales y madurativos en niños con trastorno por déficit de atención/ hiperactividad. Revista logos ciencia E tecnologia, 9(2), 27-43. https://doi.org/10.22335/rlct.v9i2.435

Zortéa, L. E., Kreutz, C. M., \& Johann, R. L. V. O. (2008). Imagem corporal em crianças institucionalizadas e em crianças não institucionalizadas. Aletheia, 27(1), 111-125. http://pepsic.bvsalud.org/scielo.php?script=sci_arttext\&pid=S1413-03942008000100009

Wechsler, S. M. (2003). DFH-III: O desenho da figura humana: Avaliação do desenvolvimento cognitivo infantil. LAMP/PUC.

Wechsler, S. M. (2011). Sistema de Triagem Emocional - DFH: guia de correção. LAMP/PUC.

Wechsler, S. M. (2018). DFH-IV. O desenho da figura humana. Avaliação do desenvolvimento cognitivo de crianças brasileiras. LAMP/PUC. 
Wechsler, S. M. \& Nakano, T.C. (2012). O desenho infantil: forma de expressão cognitiva, criativa e emocional. Casa do Psicólogo.

Wechsler, S. M., Prado, C. M., Oliveira, K. S., \& Mazzarino, B. G. (2011). Desenho da figura humana: análise de prevalência de indicadores para avaliação emocional. Psicologia: Reflexão e crítica, 24(3), 411-418. https://doi.org/10.1590/S0102-79722011000300001

\section{Sobre as autoras}

Chaielen Marchiolli Barboza é psicóloga, mestre em Psicologia pela Pontifícia Universidade Católica de Campinas (PUCCAMP). Atualmente é doutoranda em Psicologia na linha de pesquisa em "Instrumentos e Processos em Avaliação Psicológica" pela mesma Universidade, bolsista CAPES-1.

Solange Muglia Weschler é psicóloga, mestre e doutorado pela University of Georgia (EUA) e pós-doutorado pelo Torrance Center of Creative Studies. É professora da Pontifícia Universidade Católica de Campinas onde dirige o Laboratório de Avaliação e Medidas Psicológicas.

\section{Como citar este artigo}

Barboza, C. M., \& Wechsler, S. M. (2021). Evidências de validade dos indicadores emocionais no Desenho da Figura Humana. Avaliação Psicológica, 20(3), 321-330. http://dx.doi.org/10.15689/ap.2021.2003.19838.06 